\title{
Sirtuin 3 improves fatty acid metabolism in response to high nonesterified fatty acids in calf hepatocytes by modulating gene expression
}

\author{
Lei Liu, ${ }^{1}$ Dongmei Xing, ${ }^{1,2}$ Xiliang Du, ${ }^{2}$ Tao Peng, ${ }^{1}$ Joseph W. McFadden, ${ }^{3}$ Lixin Wen, ${ }^{1}$ Hongyu Lei, ${ }^{1}$ \\ Wei Dong, ${ }^{1}$ Guowen Liu, ${ }^{2}$ (D) Zhe Wang, ${ }^{2}$ Jianming Su, ${ }^{1 *} \mathrm{Jianh}$ ua $\mathrm{He}^{4}{ }^{4}$ (D) and Xinwei $\mathrm{Li}^{2 *}$ (D) \\ ${ }^{1}$ College of Veterinary Medicine, Hunan Provincial Key Laboratory of Protein Engineering in Animal Vaccines, Hunan Collaborative Innovation \\ Center for Safety Production of Livestock and Poultry, Hunan Agricultural University, Changsha 410128, China \\ ${ }^{2}$ Key Laboratory of Zoonosis Research, Ministry of Education, College of Veterinary Medicine, Jilin University, 5333 Xi'an Road, Changchun, \\ Jilin Province 130062, China \\ ${ }^{3}$ Department of Animal Science, Cornell University, Ithaca, NY 14853 \\ ${ }^{4}$ College of Animal Science and Technology, Hunan Agricultural University, Changsha 410128, China
}

\section{ABSTRACT}

Sirtuin 3 (SIRT3), a mitochondrial deacetylase, is a key regulator of energy metabolism in the liver. In nonruminants, the hepatic abundance of SIRT3 is decreased in individuals with nonalcoholic fatty liver diseases, and recovery of SIRT3 alleviates hepatic triacylglycerol (TG) deposition. However, the level of SIRT3 expression and its effects on lipid metabolism in dairy cows have not been characterized. Here we studied the hepatic expression of SIRT3 in cows with fatty liver and the role of SIRT3 in fatty acid metabolism in bovine hepatocytes. This in vivo study involved 10 healthy cows and 10 cows with fatty liver, from which we collected samples of liver tissue and blood. Primary hepatocytes were isolated from Holstein calves and treated with $0,0.5$, or $1.0 \mathrm{~m} M$ nonesterified fatty acids (NEFA) for $24 \mathrm{~h}$ or transinfected with SIRT3 overexpression adenovirus (Ad-SIRT3)/SIRT3-short interfering (si)RNA for $48 \mathrm{~h}$. Cows with fatty liver displayed lower serum glucose concentrations but higher serum NEFA and $\beta$-hydroxybutyrate concentrations relative to healthy cows. Cows with fatty liver also had significant lower mRNA and protein abundance of hepatic SIRT3. Incubation of primary hepatocytes with NEFA reduced SIRT3 abundance in primary hepatocytes in a dose-dependent manner. Fatty acid $(1 \mathrm{~m} M)$ treatment also markedly increased the abundance of acetyl-CoA carboxylase 1 (ACC1) and fatty acid synthase (FAS) but significantly decreased the abundance of carnitine

Received September 28, 2019.

Accepted March 5, 2020.

*Corresponding authors: sjmauhn@163.com and lixinwei100@126 palmitoyltransferase I $(C P T 1 A)$, carnitine palmitoyltransferase II (CPT2), and acyl-CoA oxidase (ACO). Knockdown of SIRT3 by SIRT3-siRNA downregulated the mRNA abundance of CPT1A, CPT2, and ACO. In contrast, overexpression of SIRT3 by Ad-SIRT3 upregulated the mRNA abundance of $C P T 1 A, C P T 2$, and $A C O$; downregulated the mRNA abundance of $A C C 1$ and $F A S$; and consequently, decreased intracellular TG concentrations. Overexpression of SIRT3 ameliorated exogenous NEFA-induced TG accumulation by downregulating the abundance of $A C C 1$ and $F A S$ and upregulating the abundance of CPT1A, CPT2, and $A C O$ in calf hepatocytes. Our data demonstrated that cows with fatty liver had lower hepatic SIRT3 contents, and an increase in SIRT3 abundance by overexpression mitigated TG deposition by modulating the expression of lipid metabolism genes in bovine hepatocytes. These data suggest a possible role of SIRT3 as a therapeutic target for fatty liver disease prevention in periparturient dairy cattle.

Key words: sirtuin 3 (SIRT3), nonesterified fatty acids, hepatocytes, perinatal period

\section{INTRODUCTION}

Fatty liver is one of the most common perinatal disorders in dairy cows. It is characterized by the infiltration of lipids in liver parenchyma, with triacylglycerols (TG) being the predominant lipid type. Fatty liver disease is categorized as mild (1-5\%), moderate (5-10\%), or severe $(>10 \%)$ based on hepatic TG content (wet weight). About 20 to $65 \%$ of early lactation cows experience mild or moderate fatty liver, and 5 to $24 \%$ of cows develop the severe form (Bobe et al., 2004). The development of fatty liver disease may compromise milk yield and increase a cow's susceptibility to dystocia, infection, inflammation, infertility, and early 
culling (Wensing et al., 1997; Mulligan and Doherty, 2008). Because these outcomes represent a major economic loss for dairy producers, understanding the etiology, pathology, and prevention of fatty liver would have benefits for profitability in the dairy industry.

Dairy cows undergo a period of negative energy balance during early lactation because of the increasing energy expenditure for milk production alongside decreasing DMI (Ingvartsen, 2006; Friggens et al., 2007). Negative energy balance induces fat mobilization and subsequently results in increased serum concentrations of nonesterified fatty acids (NEFA). Excessive NEFA absorbed by the liver from plasma can be re-esterified into TG and inhibit the assembly of very low density lipoprotein in hepatocytes (Liu et al., 2014), thereby inducing varying degrees of fatty liver in dairy cows.

Sirtuin 3 (SIRT3) is a member of the sirtuins, a family of $\mathrm{NAD}^{+}$-dependent histone deacetylases (Nogueiras et al., 2012), and it is predominantly located in mitochondria. It regulates lipid metabolism (Kendrick et al., 2011) and is involved in the progression of metabolic and vascular diseases, cancer, and neurodegenerative diseases (Houtkooper et al., 2012; Sosnowska et al., 2017; Kane and Sinclair, 2018). Sirtuin 3 deacetylates enzymes such as mitochondrial long-chain specific acylCoA dehydrogenase, very long-chain specific acyl-CoA dehydrogenase, enoyl-CoA hydratase, and hydroxyacylcoenzyme A dehydrogenase, and it stimulates catalytic activity to boost mitochondrial fatty acid $\beta$-oxidation (Hirschey et al., 2010; Hebert et al., 2013). Fasted SIRT3 knockout mice display abnormal lipid metabolism with an accumulation of hepatic TG, and recovery of SIRT3 restores palmitate oxidation (Hirschey et al., 2010).

Limited information exists regarding the role of SIRT3 in ruminants. The SIRT3 gene is expressed in the liver, muscle, and adipose tissue of cattle, and its hepatic expression is higher in mature cows than in calves (Ghinis-Hozumi et al., 2011). In goats, the hepatic protein abundance of SIRT3 is lower during the first 2 wk postpartum, relative to $>3$ wk prepartum (Liu et al., 2018). Additionally, serum NEFA concentrations inversely correlate with mRNA expression of hepatic SIRT3 in goats, which suggests that systemic NEFA metabolism may modulate SIRT3 functionality via changes in transcription (Liu et al., 2018). We investigated SIRT3 expression in dairy cattle with and without fatty liver and evaluated the role of SIRT3 in NEFA metabolism in calf hepatocytes. We hypothesized that the hepatic expression of SIRT3 would be lower in cows with fatty liver, and SIRT3 would regulate fatty acid metabolism in response to high NEFA treatment in calf hepatocytes.

\section{MATERIALS AND METHODS}

\section{Blood and Liver Sample Collection in Cows}

The animal study was approved and overseen by the Institutional Animal Care and Use Committees at Hunan Agricultural University and Jilin University (201711038). The animals received humane care according to the principles and specific guidelines presented in Guide for the Care and Use of Agricultural Animals in Research and Teaching (FASS, 2010). Holstein cows were studied on a commercial dairy farm with approximately 4,000 mature cows in Changchun, Jilin, China. The TMR was formulated to meet lactating cow nutritional requirements. Cows were fed ad libitum twice each day at 0830 and $1600 \mathrm{~h}$. The basal diet formulation is shown in Supplemental Table S1 (https://doi .org/10.3168/jds.2019-17670). To obtain statistically significant differences regarding the relative hepatic SIRT3 levels between healthy cows and cows with fatty liver, we calculated sample size with $P=0.01$ and power at $95 \%$ (https://www.bu.edu/researchsupport/ compliance/animal-care/working-with-animals / research/sample-size-calculations-iacuc/). We assumed that the standard deviation of relative hepatic SIRT3 levels was 0.25 , and we aimed to see a 1 -fold difference in relative SIRT3 abundance between healthy cows and cows with fatty liver. These conditions required that 9 animals be included in each group. We added an extra animal in each group in case of sample loss. Hence, 10 healthy animals and 10 cows with fatty liver were needed. Sixty cows were preselected by certified veterinarians. The cows had similar parities (median $=3$, range $=2$ to 4 ) and lactations (median $=6$ DIM, range $=4$ to 9 DIM), but their selection was otherwise random. The cows underwent routine physical examinations by experienced veterinarians to ensure no other perinatal diseases, such as hypocalcemia, mastitis, and so on, were present. The BCS was assigned based on a 1- to 5-point scale according to a previous report (Edmonson et al., 1989). Among the 60 cows, 25 cows had significantly lower DMI and milk yield but higher BCS and were suspected to have fatty liver. Liver tissue samples were obtained from the 60 cows to measure the TG content. Healthy cows were defined as having liver TG content $<1 \%(\mathrm{~g} / \mathrm{g} \times 100 \%$ of wet weight $)$; normal serum glucose, NEFA, and BHB levels; and no clinical symptoms. Cows with hepatic TG $>5 \%$ were identified as having fatty liver (Bobe et al., 2004). Twelve cows in the group suspected to have fatty liver had hepatic TG $>5 \%$. Two cows developed mastitis shortly after sampling and were thus excluded from further study. Ten cows were randomly selected from among the remain- 
Table 1. The productive and physiological variables of cows classified as healthy or fatty liver

\begin{tabular}{|c|c|c|c|c|c|}
\hline Item & \multicolumn{2}{|c|}{ Healthy cows $(\mathrm{n}=10)$} & \multicolumn{2}{|c|}{ Cows with fatty liver $(\mathrm{n}=10)$} & $P$-value \\
\hline BW (kg) & 637 & 617,659 & 662 & 634,691 & 0.05 \\
\hline DMI $(\mathrm{kg} / \mathrm{d})$ & 21.4 & $20.2,21.7$ & 20.2 & $19.3,21$ & 0.03 \\
\hline BCS & 2.79 & $2.6,2.85$ & 3.07 & $2.94,3.17$ & $<0.01$ \\
\hline Glucose $(\mathrm{m} M)$ & 3.54 & $3.01,3.97$ & 3.07 & $2.67,3.34$ & $<0.01$ \\
\hline
\end{tabular}

${ }^{1} \mathrm{NEFA}=$ nonesterified fatty acids.

ing healthy cows and used as controls. The productive and physiological parameters of the healthy and fatty liver cows are shown in Table 1.

Jugular vein blood collection was performed before feeding on 3 consecutive days between 0700 and 0800 h. Serum was harvested by centrifugation at $3,500 \times g$ for 15 min at $4^{\circ} \mathrm{C}$ immediately after blood sampling and stored at $-80^{\circ} \mathrm{C}$ for further analysis. Concentrations of glucose, BHB, and NEFA in serum were determined using a Hitachi 7170 autoanalyzer (Hitachi, Tokyo, Japan) with commercially available kits (BHB, RB1008; NEFA, FA115; glucose, GL3815; Randox Laboratories, Crumlin, UK). The limits of quantification of glucose, $\mathrm{BHB}$, and NEFA were 0.200 to $35.5 \mathrm{mM}, 0.100$ to 5.75 $\mathrm{m} M$, and 0.072 to $2.24 \mathrm{mM}$, respectively. Intra-assay coefficients of variation (CV) for glucose, BHB, and NEFA were $<3.5 \%,<5 \%$ and $<5 \%$, respectively. Interassay CV for glucose, BHB, and fatty acids were $<10 \%$, $<10 \%$, and $<15 \%$, respectively.

Liver biopsies were performed by needle puncture (Shanghai Surgical Equipment Factory, Shanghai, China) and are described in detail elsewhere (Du et al., 2018a). In brief, the intercostal space was shaved and sanitized. After cows received anesthesia by hypodermic injection of $2 \%$ lidocaine $\mathrm{HCl}$ (Sigma, St. Louis, MO), a needle was inserted through an incision of $\leq 3 \mathrm{~mm}$ at the surgical site to obtain about $100 \mathrm{mg}$ liver tissues. Tissue samples were snap-frozen in liquid nitrogen for further use within a month. Cows were transferred to a separate pen after liver biopsy, and veterinarians kept a close watch on their status.

\section{Construction of Recombinant Adenoviruses and NEFA Preparation}

The empty adenovirus vector (Ad-GFP; with green fluorescent protein), SIRT3 overexpression adenovirus (Ad-SIRT3), and short interfering (si)RNA were constructed by Hanbio (Shanghai, China). To obtain stable SIRT3 silencing, we used an equivalent mixture of 3 SIRT3 short hairpin RNA sequences. Their sequences were as follows: si-SIRT3-1, sense 5'-GGUGGAGGAUGGUCCAUAUTT-3', antisense 5'-AUAUGGACCAUCCUCCACCTT-3'; si-SIRT3-2, sense 5'-CCAGCGGCAUCCCAGACUUTT-3', antisense 5'-AAGUCUGGGAUGCCGCUGGTT-3'; and si-SIRT3-3, sense 5'-CCCUGACUCAAAGCUCGUUTT-3', antisense 5'-AACGAGCUUUGAGUCAGGGTT-3'. The titer of adenovirus before administration was $>1 \times 10^{10} \mathrm{pfu} /$ $\mathrm{mL}$. The NEFA was prepared in accordance with a previous study (Liu et al., 2014), diluted in $0.1 \mathrm{M} \mathrm{KOH}$ at $60^{\circ} \mathrm{C}$, and adjusted to $\mathrm{pH} 7.4$ with $\mathrm{HCl}(1 \mathrm{M})$. The stock NEFA $(52.7 \mathrm{mM})$ solution consisted of C16:1 (2.8 $\mathrm{m} M), \mathrm{C} 18: 1$ (22.9 mM), C18:2n-6 (2.6 mM), C16:0 (16.8 $\mathrm{m} M)$, and C18:0 (7.6 $\mathrm{mM})$. All fatty acid standards were purchased from Sigma-Aldrich (St. Louis, MO).

\section{Calf Primary Hepatocyte Isolation, Culture, and Treatment}

We adhered to the same isolation and culture protocol as previously defined by Liu et al. (2014). Hepatocytes were isolated using the collagenase IV perfusion method. Three 1-d-old Holstein female newborns (30-40 kg, healthy, fasting) were purchased from Ground Dairy Pasture (Changchun, Jilin). The caudate lobe of the liver was obtained by surgical resection. Then, the liver was perfused through vessels with perfusion solution A $(140 \mathrm{~m} M \mathrm{NaCl}, 10 \mathrm{~m} M$ HEPES, $6.7 \mathrm{~m} M \mathrm{KCl}, 0.5 \mathrm{~m} M$ EDTA, and $2.5 \mathrm{~m} M$ glucose, $\mathrm{pH}$ $7.2-7.4,37^{\circ} \mathrm{C}$ ) at a flow rate of $50 \mathrm{~mL} / \mathrm{min}$ for $12 \mathrm{~min}$, which was followed by solution B $(140 \mathrm{~m} M \mathrm{NaCl}, 30$ $\mathrm{m} M$ HEPES, $6.7 \mathrm{~m} M \mathrm{KCl}, 5 \mathrm{~m} M \mathrm{CaCl}_{2}$, and $2.5 \mathrm{~m} M$ glucose, $\mathrm{pH} 7.2-7.4,37^{\circ} \mathrm{C}$ ) at a flow rate of $50 \mathrm{~mL} /$ min for 3 min. After the outflow became transparent, collagenase IV solution ( $0.1 \mathrm{~g}$ collagenase IV dissolved in $0.5 \mathrm{~L}$ of perfusion solution $\mathrm{B}, \mathrm{pH} 7.2-7.4,37^{\circ} \mathrm{C}$ ) was introduced at a flow rate of $20 \mathrm{~mL} / \mathrm{min}$ for 15 to 20 min. The collagenase digestion was terminated by addition of $100 \mathrm{~mL}$ of fetal bovine serum (FBS; Hyclone Laboratories, Logan, UT). The liver capsule, blood vessels, fat, and connective tissues were carefully removed, 
and the remainder of the liver parenchyma was minced and filtered successively through a $150-\mu \mathrm{m}$ mesh and a $75-\mu \mathrm{m}$ mesh. Hepatocytes were then washed twice with basic medium (Hyclone Laboratories) at $4^{\circ} \mathrm{C}$ and resuspended in adherent medium (RPMI-1640 basic medium supplemented with $10 \% \mathrm{FBS}, 10^{-6} M$ insulin, $10^{-6} M$ dexamethasone, $10 \mu \mathrm{g} / \mathrm{mL}$ vitamin $\mathrm{C}$ ). Cells were seeded into a 6 -well tissue culture plate at $1 \times$ $10^{6}$ cells $/ \mathrm{mL}$ for incubation at $37^{\circ} \mathrm{C}$ and $5 \% \mathrm{CO}_{2}$. The medium was renewed to growth medium (RPMI-1640 basic medium $+10 \%$ FBS) after $24 \mathrm{~h}$.

After a 3-d incubation, hepatocytes were maintained in medium without serum for $6 \mathrm{~h}$ and treated with 0 , 0.5 , or $1 \mathrm{~m} M$ NEFA cocktail in basic medium containing $2 \%$ BSA (wt/vol) for $12 \mathrm{~h}$. Cells were harvested by using trypsin and stored at $-80^{\circ} \mathrm{C}$ for further use within 2 wk. The SIRT3-siRNA was delivered to cells by following the instruction of Lipofectamine 2000 Transfection Reagent (Invitrogen, Waltham, MA). Cells were collected after 48-h incubation. Calf hepatocytes were infected with Ad-SIRT3/empty adenovirus at different multiplicity of infection (MOI) ratios $(\mathrm{MOI}=0,25$, $50,100)$. After incubation for $48 \mathrm{~h}$, cells were harvested. To investigate the effects of SIRT3 overexpression plus high levels of NEFA, cells were transfected with AdSIRT3 for $30 \mathrm{~h}$, then serum starved for $6 \mathrm{~h}$, and finally co-incubated with $1 \mathrm{~m} M$ NEFA for $12 \mathrm{~h}$.

\section{Hepatic TG Quantification}

Liver biopsy samples (approximately $20 \mathrm{mg}$ ) were homogenized in ice-cold PBS (1:20, wt/vol). Calf hepatocytes were washed twice with ice-cold PBS. Triacylglycerol levels were determined with commercial kits by following the manual from the manufacturer (E1013; Applygen Technologies Inc., Beijing, China). The detection range of the kit was from 20 to $2,000 \mu M$.

\section{Oil Red O Staining}

Hepatocytes were fixed in $10 \%$ formalin for $20 \mathrm{~min}$. After being washed twice with ice-cold PBS, cells were incubated with $60 \%$ (vol/vol) isopropanol for $2 \mathrm{~min}$, stained with $0.5 \%$ (wt/vol) Oil Red O solution for 30 min, and washed with 60\% isopropanol and ice-cold PBS. Then, cells were counter-stained with hematoxylin before microscopy (Nikon Instruments Shanghai Co., Ltd., Shanghai, China).

\section{Quantitative Reverse-Transcription PCR Assay}

Immediately after being removed from the liquid nitrogen tank or $-80^{\circ} \mathrm{C}$ freezer, the liver biopsy samples and hepatocytes were subject to RNA extraction. Total
RNA was extracted using RNAiso Plus (D9108A, TaKaRa Biotechnology Co. Ltd., Dalian, China) according to the manufacturer's instructions. After a quality check using a K5500 MicroSpectrophotometer (Beijing Kaiao Technology Development Ltd., Beijing, China) and electrophoresis (1\% agarose gels), about $2 \mu \mathrm{g}$ of total RNA was reverse-transcribed to cDNA using a Reverse Transcription Kit (RR047A, TaKaRa Biotechnology Co. Ltd., Tokyo, Japan) according to the manufacturer's instructions. In the present study, the $\mathrm{OD}_{260} /$ $\mathrm{OD}_{280}$ ratio of the total RNA was 1.85 to 1.98 , which was within the range for RNA quality according to MIQE (Minimum Information for Publication of Quantitative Real-Time PCR Experiments) guidelines. Electrophoresis of total RNA displayed 3 bands (28S, $18 \mathrm{~S}$, and $5 \mathrm{~S}$ ). To eliminate gDNA contamination, the RNA extracts were treated with gDNA Eraser (included in the kit) for $2 \mathrm{~min}$ at $42^{\circ} \mathrm{C}$. The mRNA abundance was determined using a FastStart Universal SYBR Green Master (ROX) (4913850001, Roche, Norwalk, CT) with the 7500 Real-Time PCR System (Applied Biosystems Inc., Waltham, MA). All melt curves of SYBR-based PCR targets were singly and discretely peaked. The reaction system consisted of $10 \mu \mathrm{L}$ of SYBR Green Master, $1 \mu \mathrm{L}$ of each primer, $1 \mu \mathrm{L}$ of $\mathrm{cDNA}$, and $7 \mu \mathrm{L}$ of RNase-free distilled $\mathrm{H}_{2} \mathrm{O}$. The reaction program was as follows: initial denaturation at $95^{\circ} \mathrm{C}$ for $3 \mathrm{~min}, 40$ cycles of denaturation at $95^{\circ} \mathrm{C}$ for $15 \mathrm{~s}$, annealing at $60^{\circ} \mathrm{C}$ for $1 \mathrm{~min}$, and extension at $60^{\circ} \mathrm{C}$ for $1 \mathrm{~min}$, and a final extension at $72^{\circ} \mathrm{C}$ for $5 \mathrm{~min}$. The amplification curves showed a good dose-dependent manner upon different cDNA template concentrations, with $\mathrm{Ct}$ in the range of 12 to 25 . The calibration curves exhibited a good linear relationship with the correlation coefficient $\left(r^{2}\right)>0.994$. The PCR efficiency was consistent, ranging from 98.7 to $103.6 \%$. The cycles-to-threshold values of $A C T B$ and $G A P D H$, which were used as the internal controls, were not significantly different between treatments (Supplemental Figure S1; https://doi.org/ 10.3168/jds.2019-17670). The relative expression of target genes was normalized to $A C T B$ and $G A P D H$ and calculated with the $2^{-\Delta \Delta \mathrm{CT}}$ method. The primers were designed using the Primer-BLAST tool available from the NCBI website (https://www.ncbi.nlm .nih.gov/tools/primer-blast/) according to a previous study (Ye et al., 2012) and presented in Supplemental Table S2 (https://doi.org/10.3168/jds.2019-17670). The PCR amplification was done in triplicate for each cow. Healthy cows were used as controls, and cows with fatty liver composed the experimental group. The PCR amplification was repeated 9 times for each treatment in vitro. Hepatocytes without any treatments were used as controls, and hepatocytes with treatments were the experimental groups. 


\section{Protein Extraction and Western Blotting}

The total protein was extracted from liver tissues and calf hepatocytes using a kit (P0013B, Beyotime Institute of Biotechnology, Jiangsu, China) according to instructions. The concentration of extracted protein was determined with a BCA Protein Assay Kit (P1511, Applygen Technologies Inc.). A total of $30 \mu \mathrm{g}$ of protein from each sample was loaded into 8 to $12 \%$ Tris-glycine gels with markers (26616; Thermo Fisher Scientific, Waltham, MA). Target proteins were electroblotted onto $0.45-\mu \mathrm{m}$ polyvinylidene fluoride membranes, blocked with $3 \%$ BSA/Tris-buffered saline/Tween, and then hybridized overnight at $4{ }^{\circ} \mathrm{C}$ with antibodies against $\beta$-actin (internal controls) $(1: 5,000$, ab8226, Abcam, Cambridge, UK) and SIRT3 (1:500, sc-365175, Santa Cruz Biotechnology, Santa Cruz, CA), followed by incubation with secondary horseradish peroxidaseconjugated antibodies (1:5,000, BA1050, Boster, Wuhan, China). Immunoreactive bands were detected using an enhanced chemiluminescence solution (Pierce Biotechnology Inc., Chicago, IL) and further imaged using a protein imager (ProteinSimple, Santa Clara, $\mathrm{CA})$.

\section{Statistical Analysis}

In vivo data are presented as the mean \pm standard error of the mean and were subjected to statistical analysis with GraphPad Prism 5 (GraphPad InStat Software, San Diego, CA). The quantitative reverse transcription (qRT)-PCR and Western blot data of the in vivo studies were normally distributed and analyzed with $t$-test; other data were nonnormally distributed and analyzed with Wilcoxon signed rank test. Comparisons among groups in vitro were analyzed using 1-way ANOVA with Bonferroni correction. A $P$-value $<0.05$ was statistically significant.

\section{RESULTS}

\section{Hepatic SIRT3 Expression in Cows with Fatty Liver}

Significant differences were found in BW, milk production, DMI, BCS, and blood glucose, NEFA, and BHB levels between healthy and cows with fatty liver (Table 1). Compared with healthy cows, cows with fatty liver had higher body weights, BCS, and serum levels of NEFA and BHB, but lower milk production, DMI, and glucose levels. Liver TG contents in healthy cows were $<1 \%$ (g/g $\times 100 \%$ of wet weight), while those in cows with fatty liver were $>5 \%$ (Figure 1A). The protein abundance of hepatic SIRT3 was significantly lower in cows with fatty liver than in healthy controls $(P<$ 0.001; Figure 1B and 1C).

In vitro, the mRNA abundance of SIRT3 was lower in the $1 \mathrm{~m} M$ NEFA treatment group than in the control group $(P<0.01$; Figure 1D), and protein abundance of SIRT3 was lower in the 0.5 and $1 \mathrm{~m} M$ NEFA treatment groups $(P<0.01$ and $P<0.001$, respectively; Figure $1 \mathrm{E}$ and $\mathrm{F})$.

\section{Effects of SIRT3 Silencing on Lipid Metabolism in Primary Cultured Bovine Hepatocytes}

The si-SIRT3 administration significantly reduced both mRNA (Figure 2A) and protein abundance of SIRT3 in hepatocytes $(P<0.01$; Figure $2 \mathrm{~B}$ and $\mathrm{C})$. The mRNA abundance of key fatty acid synthesis genes acetyl-CoA carboxylase 1 (ACC1) and fatty acid synthase $(F A S)$ did not differ between the si-SIRT3 group and negative controls (cells transfected with control of small interfering RNA; Figure 2D and E). However, the mRNA abundance of key fatty acid oxidation genes carnitine palmitoyltransferase I $(C P T 1 A)$, carnitine palmitoyltransferase II (CPT2), and acyl-CoA oxidase $(A C O)$ were significantly lower in the si-SIRT3 group $(P<0.01$; Figure $2 \mathrm{~F}, \mathrm{G}$, and $\mathrm{H})$. Cellular TG contents did not change upon si-SIRT3 administration (Figure 2I).

\section{Effects of SIRT3 Overexpression on Lipid Metabolism in Hepatocytes}

Compared with Ad-GFP controls, the Ad-SIRT3 group had a significantly higher SIRT3 mRNA abundance $(P<0.001$; Figure $3 \mathrm{~A})$, and Western blot results confirmed the observation (Figure $3 \mathrm{~B}$ and $\mathrm{C}$ ). These data demonstrated that the Ad-SIRT3 used in the present study was effective in inducing SIRT3 overexpression. The mRNA abundance of $A C C 1$ and $F A S$ was lower in the Ad-SIRT3 group with MOI $100(P$ $=0.03$ and $P=0.04$, respectively; Figure $3 \mathrm{D}$ and $\mathrm{E})$. The mRNA abundance of CPT1A, CPT2, and ACO was enhanced by SIRT3 overexpression (Figure 3F, G, and $\mathrm{H})$. Triacylglycerol contents of hepatocytes in AdSIRT3 group were lower than in Ad-GFP controls $(P=$ 0.04; Figure 3I).

\section{Effects of SIRT3 Overexpression plus NEFA on Lipid Metabolism in Hepatocytes}

Primary calf hepatocytes were treated with AdGFP, $1 \mathrm{~m} M$ NEFA, and $1 \mathrm{~m} M$ NEFA + Ad-SIRT3, respectively. Compared with blank controls (no treat- 
ment), NEFA (1 $\mathrm{m} M)$ treatment significantly induced the expression of $A C C 1$ and $F A S(P=0.03$ and $P<$ 0.01, respectively; Figure $4 \mathrm{D}$ and $\mathrm{E})$, but inhibited the expression of CPT1A, CPT2, and ACO $(P<0.01, P$ $=0.02$ and $P=0.01$, respectively; Figure $4 \mathrm{~F}, \mathrm{G}$, and $\mathrm{H})$. Treatment with NEFA $(1 \mathrm{~m} M)$ resulted in higher TG deposition in calf hepatocytes (Figure 4J). Compared with $1 \mathrm{~m} M$ NEFA treatment alone, the SIRT3 mRNA and protein abundance was greater in the 1 $\mathrm{m} M$ NEFA + Ad-SIRT3 group $(P<0.01$; Figure $4 \mathrm{~A}$, $\mathrm{B}$, and $\mathrm{C}$ ). The mRNA abundance of $A C C 1$ and $F A S$ was also significantly lower in the $1 \mathrm{~m} M \mathrm{NEFA}+\mathrm{Ad}-$ SIRT3 group than in the $1 \mathrm{~m} M$ NEFA group $(P<0.01$; Figure 4D and E). Furthermore, the mRNA abundance of $C P T 1 A, C P T 2$, and $A C O$ was significantly higher in the $1 \mathrm{~m} M \mathrm{NEFA}+\mathrm{Ad}-\mathrm{SIRT} 3$ group than in the 1 m $M$ NEFA group $(P<0.001, P<0.01$, and $P<0.001$, respectively; Figure $4 \mathrm{~F}, \mathrm{G}$, and $\mathrm{H}$ ). Consequently, the cellular TG was also lower in the $1 \mathrm{~m} M \mathrm{NEFA}+\mathrm{Ad}-$ SIRT3 group than in the $1 \mathrm{~m} M$ NEFA group, and it was also supported by Oil Red O staining (Figure 4I).

\section{DISCUSSION}

In the present study, we reported lower hepatic abundance of SIRT3 in cows with fatty liver. The in vivo observation suggests the involvement of SIRT3 in the induction of hepatic lipid accumulation. The in vitro data revealed that NEFA inhibited the expression of SIRT3, which increased TG accumulation in hepatocytes. The mechanism of the remodeling role of SIRT3 on lipid metabolism appeared to involve upregulation of the expression of lipid oxidation genes and downregulation of the expression of lipogenic genes in bovine hepatocytes.

Cows with fatty liver display significant hepatic lipid metabolism abnormalities. The hepatocellular TG contents depend on the balance between lipogenesis and lipolysis, which are gene mediated. However, in the case of ruminants, the liver does not play a major role in lipogenesis (defined as fatty acid synthesis) because it is mostly responsible for gluconeogenesis with propionate and other glucose precursors as substrates.
A

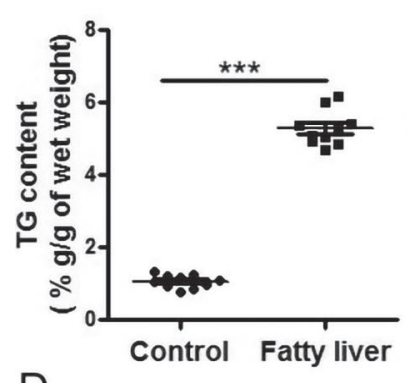

D

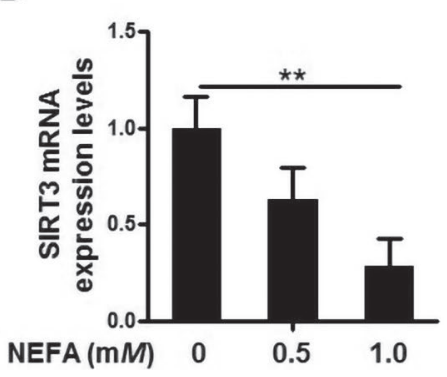

B

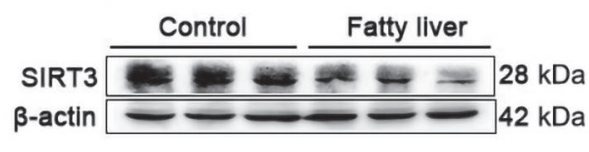

$E$

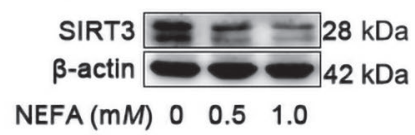

C

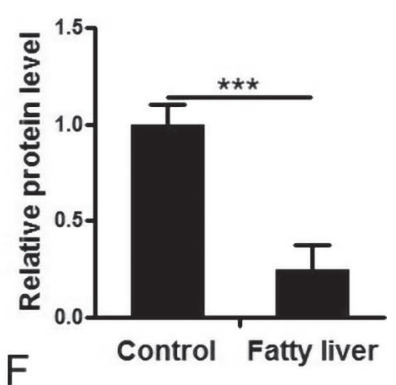

F

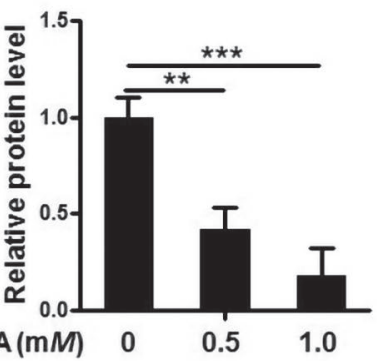

Figure 1. Hepatic sirtuin 3 (SIRT3) abundance in cows with fatty liver and effects of nonesterified fatty acids (NEFA) on expression of SIRT3 in primary calf hepatocytes. Liver biopsy samples from cows were collected and hepatic triacylglycerol (TG) contents were determined. Based on the hepatic TG levels and diagnostic evaluation by veterinarians, 10 healthy cows and 10 cows with fatty liver with similar productive parameters were enrolled. Primary calf hepatocytes were cultured, and NEFA at different concentrations were incubated with cells for $24 \mathrm{~h}$. Contents of SIRT3 in liver and hepatocytes were measured by quantitative reverse-transcription PCR (qRT-PCR) or Western blot (WB). (A) Hepatic TG contents in healthy cows and cows with fatty liver; (B) WB analysis of SIRT3 in the liver of healthy control cows and cows with fatty liver; (C) relative protein level of SIRT3 in the liver of cows; (D) mRNA expression abundance of SIRT3 in hepatocytes treated with 0, 0.5, and $1.0 \mathrm{~m} M$ NEFA; (E) WB analysis of SIRT3 in calf hepatocytes treated with 0, 0.5, and $1.0 \mathrm{~m} M$ NEFA; (F) relative protein level of SIRT3 in hepatocytes treated with $0,0.5$, and $1.0 \mathrm{~m} M$ NEFA. For all bar plots shown, data are expressed as mean \pm SEM. The qRT-PCR and Western blotting data of the in vivo studies were normally distributed and analyzed with $t$-test. Comparisons among groups in vitro were analyzed using 1-way ANOVA with Bonferroni correction. ${ }^{* *} P<0.01,{ }^{* * *} P<0.001$. 
Several proteins are involved in lipogenesis, including 2015). Prodanović et al. (2016) reported that upreguSREBP-1c, a liver isoform transcription factor control- lated expression of hepatic SREBP-1c was associated ling the de novo lipogenesis; ACC-1; and FAS ( $\mathrm{Li}$ et al., with liver lipid accumulation in dairy cows. Protein

A

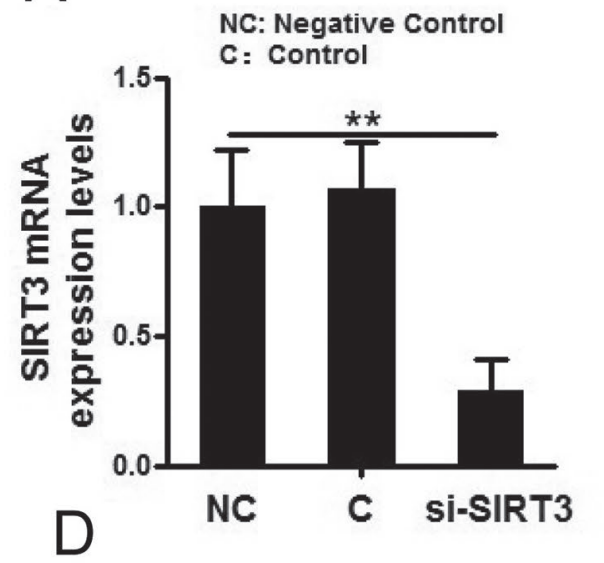

B

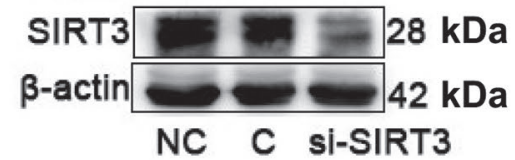

$E$
C

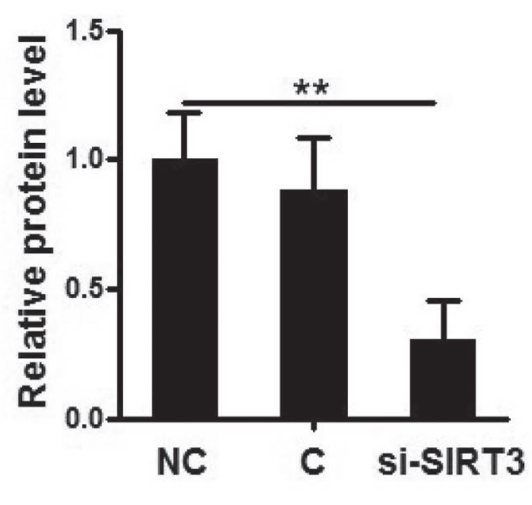

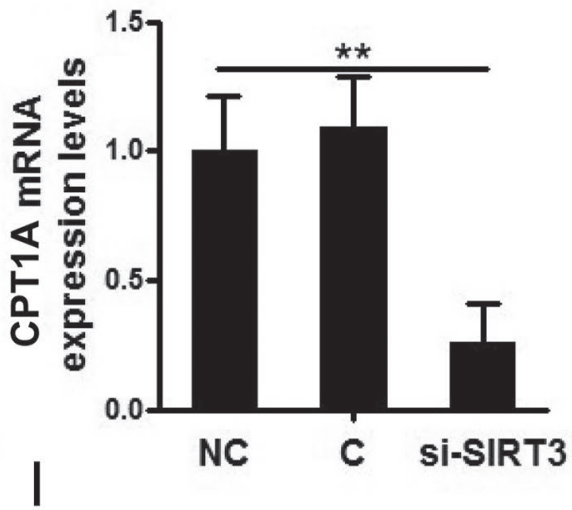

G

$\mathrm{H}$
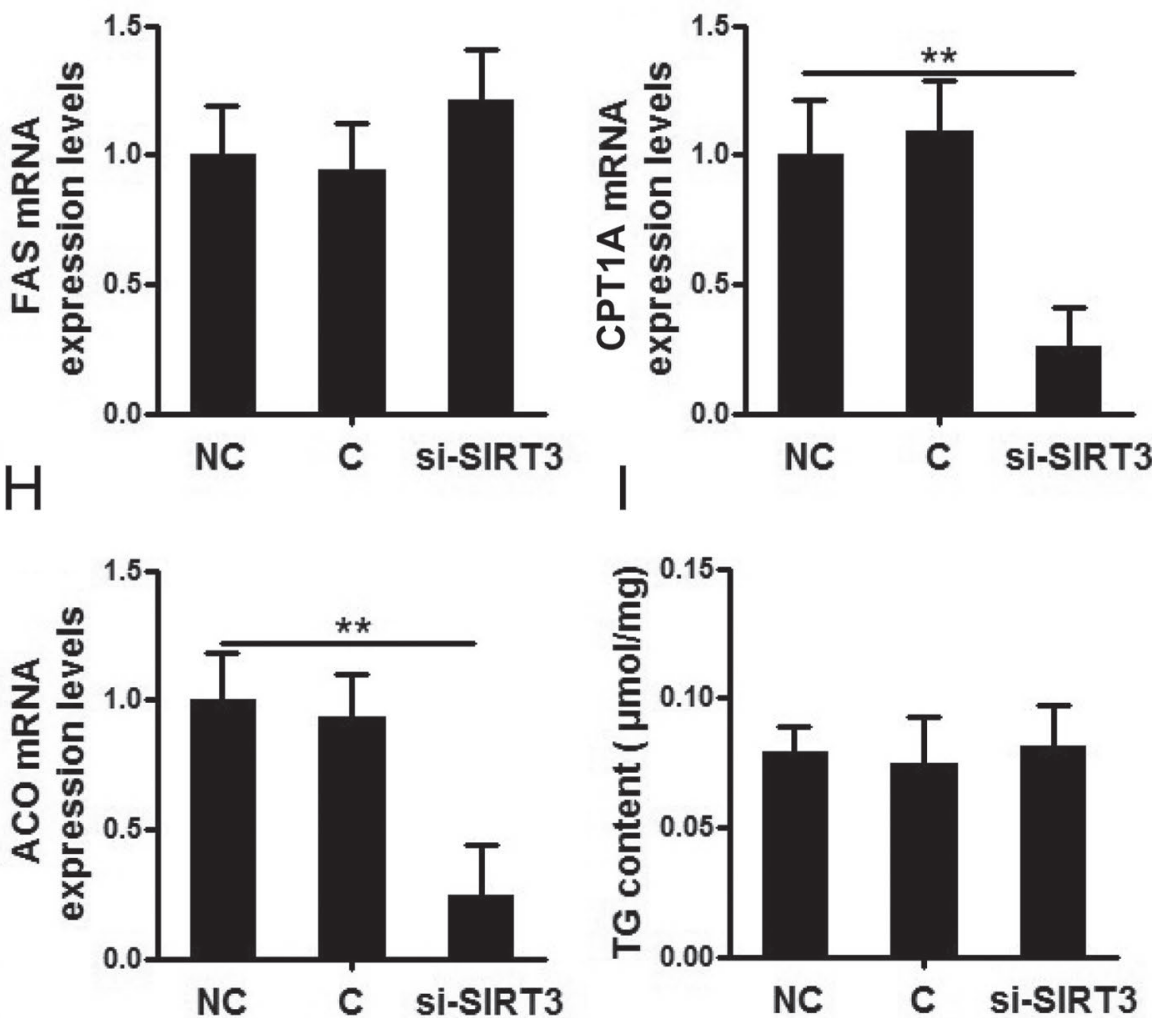

Figure 2. Effects of sirtuin 3 (SIRT3) silencing on lipid metabolism in primary calf hepatocytes. Primary calf hepatocytes were administered

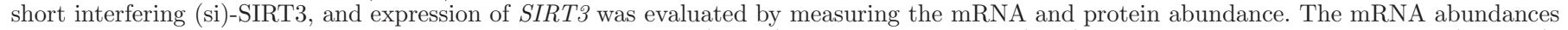
of key enzymes of lipid metabolism, acetyl-CoA carboxylase 1 ( $A C C 1)$, fatty acid synthase $(F A S)$, carnitine palmitoyltransferase I $(C P T 1 A)$, carnitine palmitoyltransferase II ( $C P$ T2), and acyl-CoA oxidase $(A C O)$, were measured by quantitative reverse-transcription PCR (qRT-PCR).

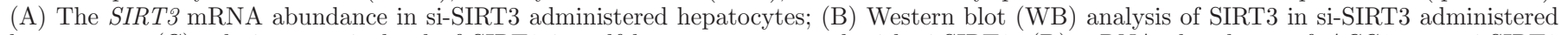
hepatocytes; (C) relative protein level of SIRT3 in calf hepatocytes treated with si-SIRT3; (D) mRNA abundance of ACC1 upon si-SIRT3

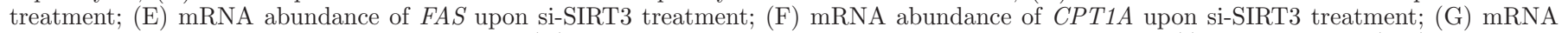
abundance of CPT2 upon si-SIRT3 treatment; $(\mathrm{H})$ mRNA abundance of $A C O$ upon si-SIRT3 treatment; (I) triacylglycerol (TG) contents in

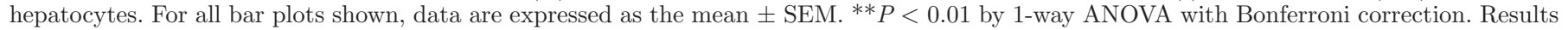
are representative of 9 independent measurements. 
A

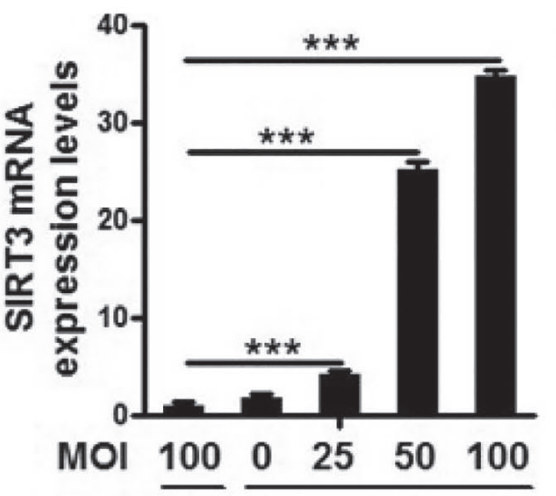

D Ad-GFP Ad-SIRT3
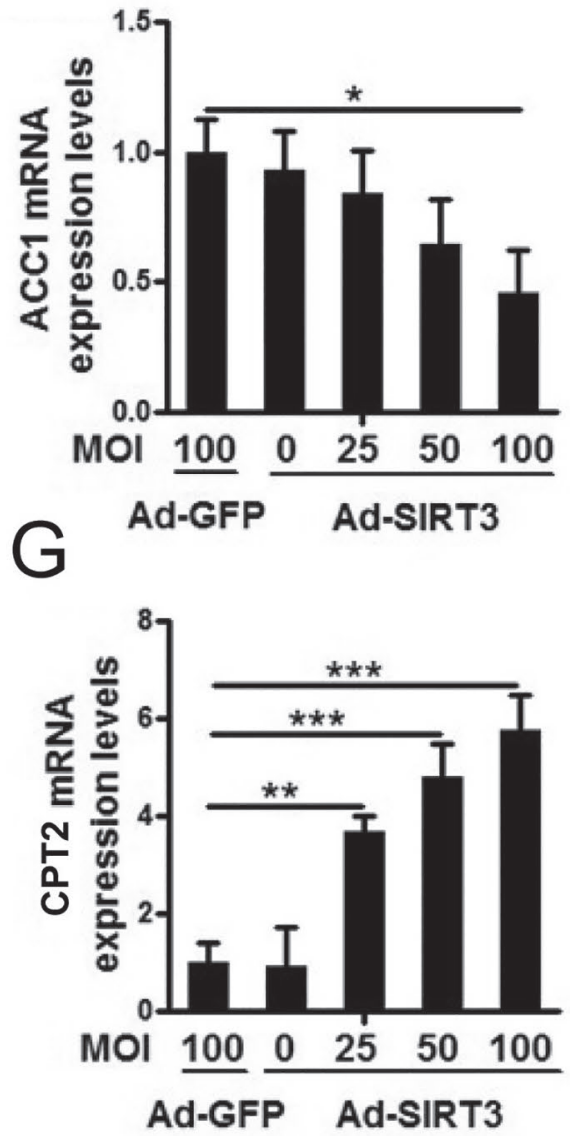

$\mathrm{B}$

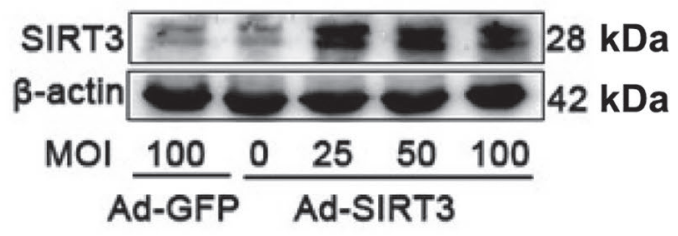

E
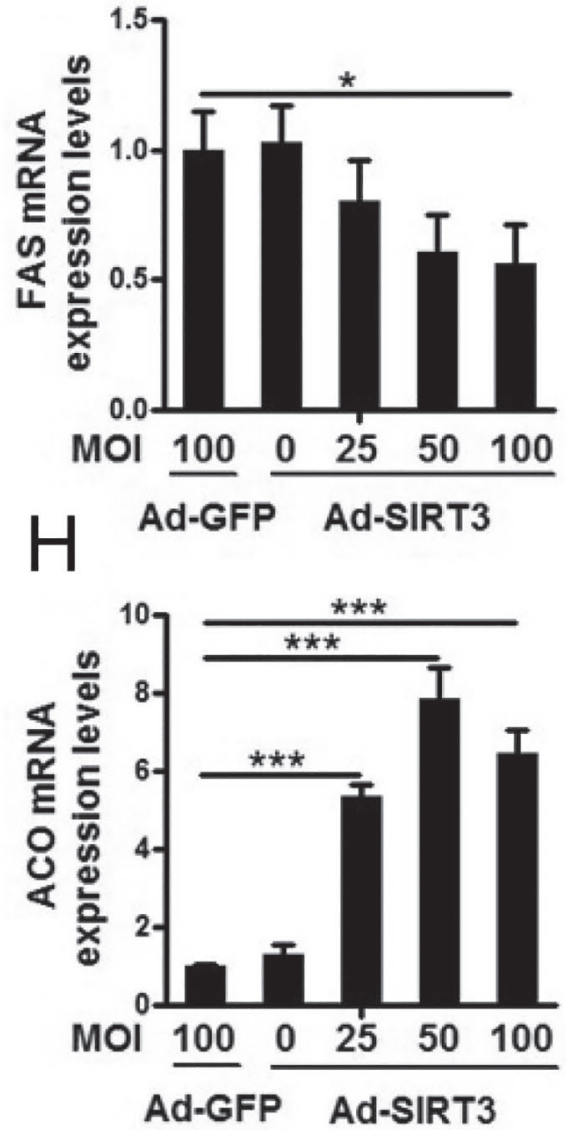

C
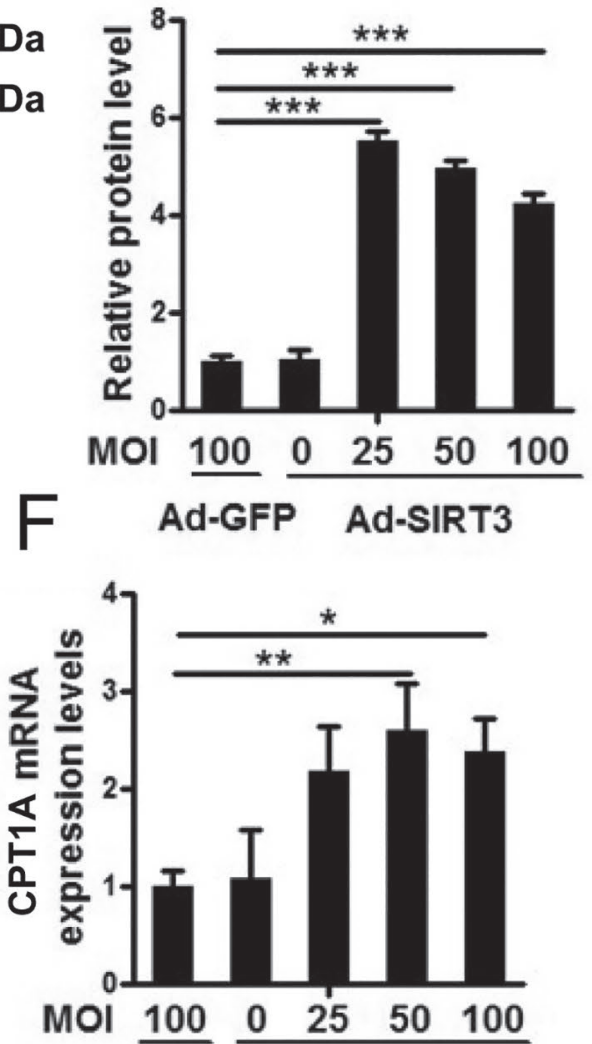

Ad-GFP Ad-SIRT3

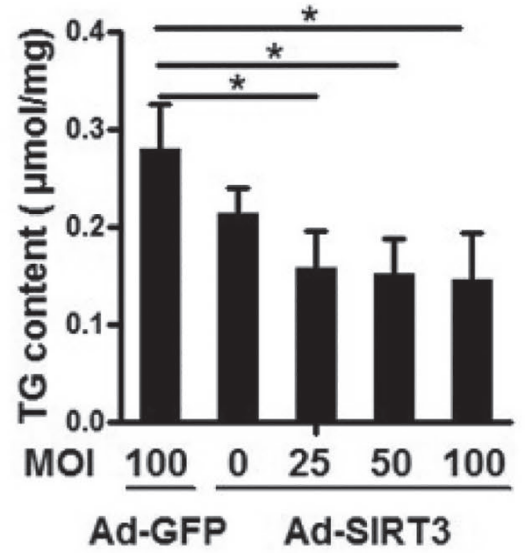

Figure 3. Effects of sirtuin 3 (SIRT3) overexpression on lipid metabolism in primary calf hepatocytes. Primary calf hepatocytes were treated with Ad-GFP (control, empty adenovirus vector with green fluorescent protein) and Ad-SIRT3, respectively, and expression of SIRT3 was estimated by measuring the mRNA and protein abundance. The mRNA abundances of key enzymes of lipid metabolism, acetyl-CoA carboxylase 1 (ACC1), fatty acid synthase $(F A S)$, carnitine palmitoyltransferase I (CPT1A), carnitine palmitoyltransferase II (CPT2), and acyl-CoA oxidase $(A C O)$, were measured by quantitative reverse-transcription PCR (qRT-PCR). (A) SIRT3 mRNA abundance in Ad-SIRT3 administered hepatocytes; (B) Western blot analysis of SIRT3 in Ad-SIRT3 administered hepatocytes; (C) relative protein level of SIRT3 in Ad-SIRT3 administered hepatocytes; (D) mRNA abundance of $A C C 1$ upon Ad-SIRT3 treatment; (E) mRNA abundance of FAS upon Ad-SIRT3 treatment; (F) mRNA abundance of CPT1A upon Ad-SIRT3 treatment; $(\mathrm{G})$ mRNA abundance of CPT2 upon Ad-SIRT3 treatment; (H) mRNA abundance of ACO upon Ad-SIRT3 treatment; (I) triacylglycerol (TG) contents in hepatocytes. For all bar plots shown, data are expressed as the mean \pm SEM. ${ }^{*} P<0.05 ;{ }^{* *} P<0.01 ;{ }^{* * *} P<0.001$ by 1 -way ANOVA with Bonferroni correction. Results are representative of 9 independent measurements. $\mathrm{MOI}=$ multiplicity of infection. 

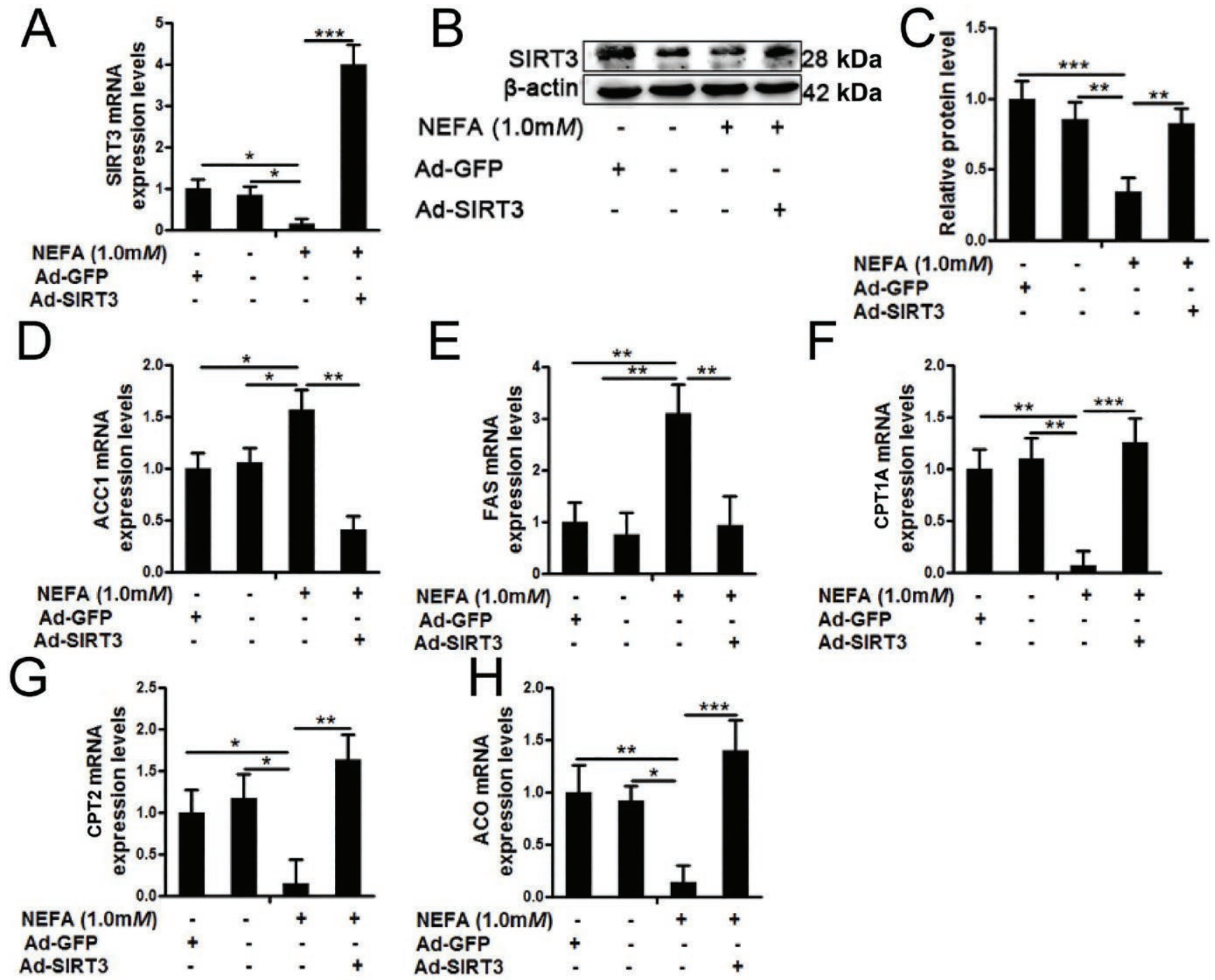

E
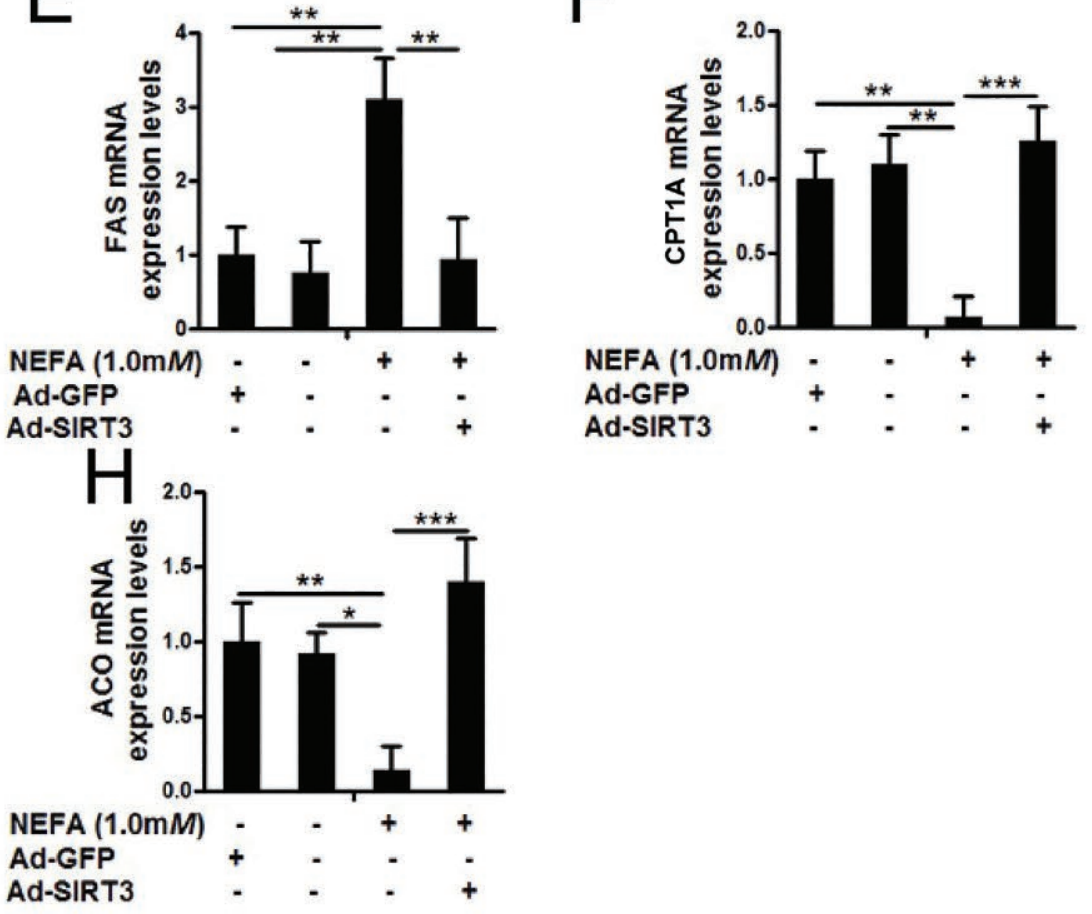

Ad-SIRT3
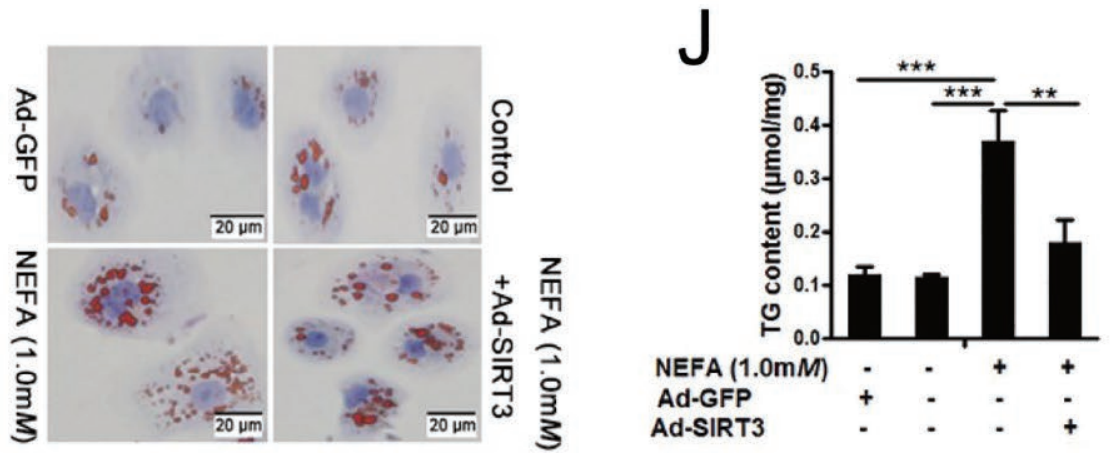

Figure 4. Effects of sirtuin 3 (SIRT3) overexpression plus nonesterified fatty acids (NEFA) on lipid metabolism in primary calf hepatocytes. Primary calf hepatocytes were treated with Ad-GFP (empty adenovirus vector with green fluorescent protein), $1 \mathrm{~m} M$ NEFA, $1 \mathrm{~m} M$ NEFA + AdSIRT3, or nothing. SIRT3 expression was studied by quantitative reverse-transcription PCR (qRT-PCR) and Western blot (WB). The mRNA abundances of acetyl-CoA carboxylase 1 ( $A C C 1)$, fatty acid synthase $(F A S)$, carnitine palmitoyltransferase I $(C P T 1 A)$, carnitine palmitoyltransferase II (CPT2), and acyl-CoA oxidase $(A C O)$ were measured by qRT-PCR. Cellular triacylglycerol (TG) contents were measured by Oil Red O staining and kits. (A) SIRT3 mRNA abundance in calf hepatocytes; (B) WB analysis of SIRT3 in calf hepatocytes; (C) relative protein abundance of SIRT3 in calf hepatocytes: (D) mRNA abundance of $A C C 1$ in calf hepatocytes; (E) mRNA abundance of FAS in calf hepatocytes; (F) mRNA abundance of CPT1A in calf hepatocytes; (G) mRNA abundance of CPT2 in calf hepatocytes; (H) mRNA abundance of $A C O$ in calf hepatocytes; (I) Oil Red O staining of calf hepatocytes, where red staining in the calf hepatocytes indicates cellular TG contents (scale bar $20 \mu \mathrm{m}) ;(\mathrm{J})$ TG contents in hepatocytes measured by kits. For all bar plots shown, data are expressed as the mean \pm SEM. ${ }^{*} P<0.05 ; * * P<$ $0.01 ; * * * P<0.001$ by 1 -way ANOVA with Bonferroni correction. Results are representative of 9 independent measurements. 
abundance of hepatic SREBP-1c and its target molecules ACC-1 and FAS increased significantly in cows with moderate fatty liver and cows with severe fatty liver (Li et al., 2015; Jia et al., 2019). It is plausible that during the development of fatty liver, the bovine lipogenesis capability is upregulated, but whether this increase can be translated into an increase in fatty acids synthesized from acetate is questionable. The contribution of the upregulation to fatty liver is undetermined. More reasonably, the regulated expression of ACC will likely lead to production of more malonyl-CoA, which serves as an inhibitor of CPT1, a key enzyme in fatty acid oxidation. Given the essential role of the liver in fatty acid utilization, hepatic oxidation capability is key to the onset of fatty liver. In addition, CPT1, CPT2 and ACO are also highly important enzymes in fatty acid oxidation. Our group previously showed that the mRNA abundance of $C P T 1 A$ and $A C O$ was upregulated in cows with mild fatty liver, indicating a compensatory mechanism (Du et al., 2018b). Others reported cows with fatty liver had significantly lower hepatic CPT activity compared with healthy cows (Mizutani et al., 1999). Thus, the abnormality of fatty acid metabolism, especially the impaired oxidation capability, further facilitates lipid deposition in the liver, leading to the deterioration of fatty liver.

Cows with fatty liver possess high serum NEFA levels, and high NEFA levels are regarded as the pathological basis for the disease. High levels of NEFA can impair hepatic lipid metabolism via disturbing gene expression in hepatocytes (Liu et al., 2014). In this study, high levels of NEFA increased the mRNA abundance of $A C C 1$ and $F A S$ and decreased the mRNA abundance of $C P T 1 A, C P T 2$, and $A C O$, suggesting that high levels of NEFA promoted lipid synthesis and impaired oxidation in hepatocytes. The imbalance between the synthesis and oxidation of fatty acids further induced TG accumulation in calf hepatocytes. This observation was consistent with previous studies in which incubation of high levels of NEFA in calf hepatocytes triggered significant TG deposition by inducing expression of $F A S$ and $A C C 1$ (Li et al., 2015; Jia et al., 2019).

Several functional molecules of energy metabolism, such as SIRT3, remodel the progression of hepatic lipid metabolism (Ding et al., 2017). Recently, findings from humans and mice with fatty liver indicated that lower hepatic SIRT3 played a crucial role in the development of fatty liver and mediated the intracellular TG accumulation induced by excessive fatty acids (Borengasser et al., 2011; Teodoro et al., 2013; Wu et al., 2014). Importantly, Liu et al. (2018) reported that hepatic SIRT3 abundance was decreased during the first $2 \mathrm{wk}$ postpartum and was negatively related to serum NEFA levels in goats. In this study, cows with moderate fatty liver also had decreased abundance of hepatic SIRT3. In vitro, a high concentration of NEFA markedly downregulated the abundance of SIRT3 in calf hepatocytes. These results indicate that low expression of SIRT3 may mediate, at least in part, the NEFA-induced expression of lipid synthesis genes and NEFA-inhibited expression of lipid oxidation genes, thereby inducing hepatic steatosis in dairy cows.

Lower hepatic abundance of SIRT3 may deteriorate fatty liver in dairy cows. To further investigate the role of SIRT3 in lipid metabolism, we transfected calf hepatocytes with si-SIRT3 or Ad-SIRT3 to inhibit or increase SIRT3 expression. We found that SIRT3 silencing restrained the expression of $C P T 1 A, C P T 2$, and $A C O$, which might have further reduced fatty acid oxidation. In contrast, overexpression of SIRT3 significantly induced expression of $C P T 1 A, C P T 2$, and $A C O$, but inhibited expression of $A C C 1$ and FAS. The gene expression profile involved in lipid metabolism upon SIRT3 overexpression favored fatty acid oxidation but limited fatty acid synthesis. Consequently, TG content in calf hepatocytes was significantly lower following AdSIRT3 administration. The mRNA abundance of $A C C 1$ and FAS did not differ between si-SIRT3 treatment and controls. This observation might be not surprising given the lack of capability of bovine hepatocytes to synthesize fatty acids. However, SIRT3 silencing did not significantly increase the cellular TG content as we had expected. This result indicates that downregulation of the key fatty acid oxidation genes may be insufficient to change cellular TG contents.

Next, we investigated the role of SIRT3 in fatty acid metabolism in the context of high NEFA. Generally, SIRT3 tended to restore lipid metabolism normality in cells treated with a NEFA cocktail. Compared with NEFA treatment alone, SIRT3 overexpression significantly downregulated the NEFA-induced mRNA levels of $A C C 1$ and $F A S$ but upregulated the mRNA levels of $C P T 1 A, C P T 2$, and $A C O$. As shown both qualitatively and quantitatively, SIRT3 overexpression attenuated the NEFA-induced TG accumulation in calf hepatocytes. This finding is important because our observation lays the foundation for further in vivo studies to investigate the availability of SIRT3 in the treatment of fatty liver in cows. A few investigations on mitigation of fatty liver diseases have already targeted SIRT3 in mice (He et al., 2013; Cheng et al., 2016; Tong et al., 2016; Li et al., 2018).

High levels of NEFA disrupt lipid metabolism in calf hepatocytes, and SIRT3 seems to serve as an antagonist to NEFA. As the level of NEFA frequently increases during the postpartum period, SIRT3 may play a broader role not only in fatty liver but also in other energy metabolism disorders in perinatal cows. 


\section{CONCLUSIONS}

Cows with fatty liver have a lower hepatic abundance of SIRT3. Knockdown of SIRT3 by si-SIRT3 significantly downregulated the mRNA abundance of CPT1A, CPT2, and ACO in calf hepatocytes. However, SIRT3 overexpression significantly induced expression of CPT1A, CPT2, and $A C O$, while inhibiting expression of $A C C 1$ and $F A S$ and consequently reducing intracellular TG content. Moreover, overexpression of SIRT3 attenuated exogenous NEFA-induced TG accumulation by downregulating the mRNA abundance of $A C C 1$ and $F A S$ and upregulating the mRNA abundance of $C P T 1 A, C P T 2$, and $A C O$. These in vitro data suggest a possible role of SIRT3 on the remodulation of lipid metabolism to normality in the event of high NEFA. Thus, we conclude that lower expression of hepatic SIRT3 influences the progression of fatty liver development during the postpartum period. Targeting this protein may lead to therapeutic applications in the management of periparturient cows. Further investigations are needed to test the feasibility of SIRT3 as a target for fatty liver treatment.

\section{ACKNOWLEDGMENTS}

The project was financially supported by China Postdoctoral Science Foundation (No. 2017M612561), the National Natural Science Foundation of China (No. 31502135), Research Foundation of Education Bureau of Hunan Province (No. 16B124), and Scientific Research Foundation for the introduction of talent in Hunan Agricultural University (15YJ05). The authors have not stated any conflicts of interest.

\section{REFERENCES}

Bobe, G., J. W. Young, and D. C. Beitz. 2004. Invited review: Pathology, etiology, prevention, and treatment of fatty liver in dairy cows. J. Dairy Sci. 87:3105-3124. https://doi.org/10.3168/jds .S0022-0302(04)73446-3.

Borengasser, S. J., F. Lau, P. Kang, M. L. Blackburn, M. J. J. Ronis, T. M. Badger, and K. Shankar. 2011. Maternal obesity during gestation impairs fatty acid oxidation and mitochondrial SIRT3 expression in rat offspring at weaning. PLoS One 6:e24068. https: //doi.org/10.1371/journal.pone.0024068.

Cheng, Y., J. Mai, T. Hou, and J. Ping. 2016. MicroRNA-421 induces hepatic mitochondrial dysfunction in non-alcoholic fatty liver disease mice by inhibiting sirtuin 3. Biochem. Biophys. Res. Commun. 474:57-63. https://doi.org/10.1016/j.bbrc.2016.04.065.

Ding, R. B., J. Bao, and C. X. Deng. 2017. Emerging roles of SIRT1 in fatty liver diseases. Int. J. Biol. Sci. 13:852-867. https://doi.org/ 10.7150/ijbs.19370.

Du, X., G. Liu, J. J. Loor, Z. Fang, R. Bucktrout, Y. Yang, Q. Ye, Z. Shi, T. Shen, X. Wang, Z. Peng, C. Zhao, B. Lv, D. Xing, Y. Zhu, X. Li, and X. Li. 2018a. Impaired hepatic autophagic activity in dairy cows with severe fatty liver is associated with inflammation and reduced liver function. J. Dairy Sci. 101:11175-11185. https:/ /doi.org/10.3168/jds.2018-15120.
Du, X., T. Shen, H. Wang, X. Qin, D. Xing, Q. Ye, Z. Shi, Z. Fang, Y. Zhu, Y. Yang, Z. Peng, C. Zhao, B. Lv, X. Li, G. Liu, and X. Li. 2018b. Adaptations of hepatic lipid metabolism and mitochondria in dairy cows with mild fatty liver. J. Dairy Sci. 101:9544-9558. https://doi.org/10.3168/jds.2018-14546.

Edmonson, A. J., I. J. Lean, L. D. Weaver, T. Farver, and G. Webster. 1989. A body condition scoring chart for Holstein dairy cows. J. Dairy Sci. 72:68-78. https://doi.org/10.3168/jds.S0022 $-0302(89) 79081-0$

FASS. 2010. Guide for the Care and Use of Agricultural Animals in Research and Teaching. 3rd ed. FASS Inc., Champaign, IL.

Friggens, N. C., C. Ridder, and P. Lovendahl. 2007. On the use of milk composition measures to predict the energy balance of dairy cows. J. Dairy Sci. 90:5453-5467. https://doi.org/10.3168/jds.2006-821.

Ghinis-Hozumi, Y., A. Gonzalez-Gallardo, L. Gonzalez-Davalos, A. Antaramian, F. Villarroya, A. Shimada, A. Varela-Echavarria, and O. Mora. 2011. Bovine sirtuins: Initial characterization and expression of sirtuins 1 and 3 in liver, muscle, and adipose tissue. J. Anim. Sci. 89:2529-2536. https://doi.org/10.2527/jas.2010-3476.

He, J., B. Hu, X. Shi, E. R. Weidert, P. Lu, M. Xu, M. Huang, E. E. Kelley, and W. Xie. 2013. Activation of the aryl hydrocarbon receptor sensitizes mice to nonalcoholic steatohepatitis by deactivating mitochondrial sirtuin deacetylase Sirt3. Mol. Cell. Biol. 33:2047-2055. https://doi.org/10.1128/MCB.01658-12.

Hebert, A. S., K. E. Dittenhafer-Reed, W. Yu, D. J. Bailey, E. S. Selen, M. D. Boersma, J. J. Carson, M. Tonelli, A. J. Balloon, A. J. Higbee, M. S. Westphall, D. J. Pagliarini, T. A. Prolla, F. Assadi-Porter, S. Roy, J. M. Denu, and J. J. Coon. 2013. Calorie restriction and SIRT3 trigger global reprogramming of the mitochondrial protein acetylome. Mol. Cell 49:186-199. https://doi .org/10.1016/j.molcel.2012.10.024.

Hirschey, M. D., T. Shimazu, E. Goetzman, E. Jing, B. Schwer, D. B. Lombard, C. A. Grueter, C. Harris, S. Biddinger, O. R. Ilkayeva, R. D. Stevens, Y. Li, A. K. Saha, N. B. Ruderman, J. R. Bain, C. B. Newgard, R. V. Farese Jr., F. Alt, C. R. Kahn, and E. Verdin. 2010. SIRT3 regulates mitochondrial fatty-acid oxidation by reversible enzyme deacetylation. Nature 464:121-125. https://doi .org/10.1038/nature08778.

Houtkooper, R. H., E. Pirinen, and J. Auwerx. 2012. Sirtuins as regulators of metabolism and healthspan. Nat. Rev. Mol. Cell Biol. 13:225-238. https://doi.org/10.1038/nrm3293.

Ingvartsen, K. L. 2006. Feeding-and management-related diseases in the transition cow: Physiological adaptations around calving and strategies to reduce feeding-related diseases. Anim. Feed Sci. Technol. 126:175-213. https://doi.org/10.1016/j.anifeedsci.2005 .08 .003 .

Jia, H., X. Li, G. Liu, J. J. Loor, R. Bucktrout, X. Sun, G. Li, X. Shu, J. Dong, Y. Wang, R. Zuo, Z. Wang, and X. Li. 2019. Perilipin 5 promotes hepatic steatosis in dairy cows through increasing lipid synthesis and decreasing very low density lipoprotein assembly. J. Dairy Sci. 102:833-845. https://doi.org/10.3168/jds.2018-15208.

Kane, A. E., and D. A. Sinclair. 2018. Sirtuins and NAD(+) in the development and treatment of metabolic and cardiovascular diseases. Circ. Res. 123:868-885. https://doi.org/10.1161/CIRCRESAHA .118 .312498 .

Kendrick, A. A., M. Choudhury, S. M. Rahman, C. E. McCurdy, M. Friederich, J. L. K. Van Hove, P. A. Watson, N. Birdsey, J. J. Bao, D. Gius, M. N. Sack, E. X. Jing, C. R. Kahn, J. E. Friedman, and K. R. Jonscher. 2011. Fatty liver is associated with reduced SIRT3 activity and mitochondrial protein hyperacetylation. Biochem. J. 433:505-514. https://doi.org/10.1042/BJ20100791.

Li, R., T. Xin, D. Li, C. Wang, H. Zhu, and H. Zhou. 2018. Therapeutic effect of Sirtuin 3 on ameliorating nonalcoholic fatty liver disease: The role of the ERK-CREB pathway and Bnip3-mediated mitophagy. Redox Biol. 18:229-243. https://doi.org/10.1016/j .redox.2018.07.011.

Li, X., W. Huang, J. Gu, X. Du, L. Lei, X. Yuan, G. Sun, Z. Wang, X. Li, and G. Liu. 2015. SREBP-1c overactivates ROS-mediated hepatic NF- $\kappa B$ inflammatory pathway in dairy cows with fatty liver. Cell. Signal. 27:2099-2109. https://doi.org/10.1016/j.cellsig .2015.07.011. 
Liu, L., X. Li, Y. Li, Y. Guan, Y. Song, L. Yin, H. Chen, L. Lei, J. Liu, X. Li, Z. Wang, X. Yang, and G. Liu. 2014. Effects of nonesterified fatty acids on the synthesis and assembly of very low density lipoprotein in bovine hepatocytes in vitro. J. Dairy Sci. 97:1328-1335. https://doi.org/10.3168/jds.2013-6654.

Liu, L., L. Yao, T. Peng, L. Wen, W. Cai, X. Jia, and J. He. 2018. Hepatic Sirt3 expression declines postpartum in dairy goats. J. Dairy Res. 85:163-166. https://doi.org/10.1017/S0022029918000171.

Mizutani, H., T. Sako, Y. Toyoda, T. Kawabata, N. Urumuhang, H. Koyama, and S. Motoyoshi. 1999. Preliminary studies on hepatic carnitine palmitoyltransferase in dairy cattle with or without fatty liver. Vet. Res. Commun. 23:475-480. https://doi.org/10.1023/A: 1006358222037 .

Mulligan, F., and M. Doherty. 2008. Production diseases: A major health, welfare and economic problem on dairy farms. Vet. J. 176:1-2. https://doi.org/10.1016/j.tvjl.2007.12.006.

Nogueiras, R., K. M. Habegger, N. Chaudhary, B. Finan, A. S. Banks, M. O. Dietrich, T. L. Horvath, D. A. Sinclair, P. T. Pfluger, and M. H. Tschop. 2012. Sirtuin 1 and sirtuin 3: Physiological modulators of metabolism. Physiol. Rev. 92:1479-1514. https://doi.org/10 .1152 /physrev.00022.2011.

Prodanović, R., G. Korićanac, I. Vujanac, A. Djordjević, M. Pantelić, S. Romić, Z. Stanimirović, and D. Kirovski. 2016. Obesity-driven prepartal hepatic lipid accumulation in dairy cows is associated with increased CD36 and SREBP-1 expression. Res. Vet. Sci. 107:16-19. https://doi.org/10.1016/j.rvsc.2016.04.007.

Sosnowska, B., M. Mazidi, P. Penson, A. Gluba-Brzozka, J. Rysz, and M. Banach. 2017. The sirtuin family members SIRT1, SIRT3 and SIRT6: Their role in vascular biology and atherogenesis. Atherosclerosis 265:275-282. https://doi.org/10.1016/j.atherosclerosis .2017 .08 .027
Teodoro, J. S., F. V. Duarte, A. P. Gomes, A. T. Varela, F. M. Peixoto, A. P. Rolo, and C. M. Palmeira. 2013. Berberine reverts hepatic mitochondrial dysfunction in high-fat fed rats: A possible role for SirT3 activation. Mitochondrion 13:637-646. https://doi .org/10.1016/j.mito.2013.09.002.

Tong, W., L. Ju, M. Qiu, Q. Xie, Y. Chen, W. Shen, W. Sun, W. Wang, and J. Tian. 2016. Liraglutide ameliorates non-alcoholic fatty liver disease by enhancing mitochondrial architecture and promoting autophagy through the SIRT1/SIRT3-FOXO3a pathway. Hepatol. Res. 46:933-943. https://doi.org/10.1111/hepr.12634.

Wensing, T., T. Kruip, M. J. H. Geelen, G. H. Wentink, and A. M. van den Top. 1997. Postpartum fatty liver in high-producing dairy cows in practice and in animal studies. The connection with health, production and reproduction problems. Comp. Haematol. Int. 7:167-171. https://doi.org/10.1007/BF02652596.

Wu, T., Y. Liu, Y. Fu, X. Liu, and X. Zhou. 2014. Direct evidence of sirtuin downregulation in the liver of non-alcoholic fatty liver disease patients. Ann. Clin. Lab. Sci. 44:410-418.

Ye, J., G. Coulouris, I. Zaretskaya, I. Cutcutache, S. Rozen, and T. L. Madden. 2012. Primer-BLAST: A tool to design target-specific primers for polymerase chain reaction. BMC Bioinformatics 13:134. https://doi.org/10.1186/1471-2105-13-134.

\section{ORCIDS}

Guowen Liu (i) https://orcid.org/0000-0002-2672-6104

Jianhua He @ https://orcid.org/0000-0002-9289-4405

Xinwei Li ๑ https://orcid.org/0000-0002-0765-677X 